\title{
Electrophoretic Retardation of Colloidal Particles in Nonpolar Liquids
}

\author{
Filip Strubbe, ${ }^{*}$ Filip Beunis, Toon Brans, Masoumeh Karvar, Wouter Woestenborghs, and Kristiaan Neyts \\ Electronics and Information Systems, Ghent University, Sint-Pietersnieuwstraat 41, Ghent B-9000, Belgium \\ and Center for Nano and Biophotonics (NB-Photonics), Ghent University, Sint-Pietersnieuwstraat 41, Ghent B-9000, Belgium
}

(Received 17 June 2011; revised manuscript received 23 November 2012; published 11 April 2013)

\begin{abstract}
We have measured the electrophoretic mobility of single, optically trapped colloidal particles, while gradually depleting the co-ions and counterions in the liquid around the particle by applying a dc voltage. This is achieved in a nonpolar liquid, where charged reverse micelles act as co-ions and counterions. By increasing the dc voltage, the mobility first increases when the concentrations of co-ions and counterions near the particle start to decrease. At sufficiently high dc voltage (around $2 \mathrm{~V}$ ), the mobility reaches a saturation value when the co-ions and counterions are fully separated. The increase in mobility is larger when the equilibrium ionic strength is higher. The dependence of the experimental data on the equilibrium ionic strength and on the applied voltage is in good agreement with the standard theory of electrophoretic retardation, assuming that the bare particle charge remains constant. This method is useful for studying the electrophoretic retardation effect and charging mechanisms for nonpolar colloids, and it sheds light on previously unexplained particle acceleration in electronic ink devices.
\end{abstract}

DOI: 10.1103/PhysRevX.3.021001

Subject Areas: Soft Matter

\section{INTRODUCTION}

In the standard theory for electrophoresis, the electrophoretic mobility of a colloidal particle results from the electrostatic force acting on the bare charge of the particle, the hydrodynamic friction force at the particle-liquid interface, and a retardation force, which is caused by the presence of a diffuse double layer around the particle [1]. On a microscopic level, the retardation force originates from the electrostatic force acting on charges in the diffuse part of the double layer. Since the double layer has a net charge that is the opposite of the bare particle charge, the volume force on this net charge generates a liquid flow that, in turn, reduces the electrophoretic mobility of the particle by hydrodynamic friction [2].

Simple expressions for the particle mobility taking into account the retardation effect, for large or thin double layers compared to the particle size, have been derived, respectively, by Hückel [3] and von Smoluchowski [4]. These expressions are obtained by solving the NavierStokes equations, by assuming a spherical, symmetrical double layer and sufficiently low surface potentials such that the Poisson-Boltzmann equation can be linearized. A more general expression for intermediate cases of the double-layer thickness was obtained by Henry [5]. More general solutions of the coupled Navier-Stokes and Poisson-Nernst-Planck equations can be obtained by using numerical calculations $[6,7]$. These results show that, for

\footnotetext{
*Corresponding author. filip.strubbe@elis.ugent.be

Published by the American Physical Society under the terms of the Creative Commons Attribution 3.0 License. Further distribution of this work must maintain attribution to the author(s) and the published article's title, journal citation, and DOI.
}

particles with high surface potentials, the relaxation effect, which takes the deformation of the double layer into account, becomes increasingly important, especially when the double-layer thickness is of the same order as the particle size. Depending on the type of particles and liquid, additional theories have been developed to include, for example, surface conduction, Helmholtz layers, and soft layers on particles [8,9]. More recently, research in this field has focused on induced-charge electrokinetic phenomena for microfluidics applications [10] and interparticle or atomic force measurements to probe the double layer [11-15].

Even though the theory of electrophoretic retardation is generally accepted, direct measurements of the retardation force are difficult and scarce. In most cases, the electrophoretic mobility is the measured quantity, and either the standard theory or indirect chemical methods such as titration are used to estimate the bare particle charge, zeta potential, and retardation force. One way of eliminating the effect of the diffuse double layer on the particle motion, and, in such a way, measuring the retardation force, is by applying large electric fields, around $10 \mathrm{~V} / \mu \mathrm{m}$ in polar media; this is referred to as the StotzWien effect [16]. In this case, the co-ions and counterions move sufficiently fast, with respect to the particle, to prevent the formation of the double layer. As a result, the retardation force disappears, and the electrophoretic mobility increases correspondingly. This nonlinear electrokinetic effect at high electric fields is observed for electrolytes (the first Wien effect) [17-19], colloids in polar liquids [20,21], and colloids in nonpolar liquids [22-24].

In this study, however, the retarding effect of the diffuse double layer is eliminated, not by applying extremely large electric fields, but by depleting the charge carriers in the 
background electrolyte with a relatively small electric field of about $0.01 \mathrm{~V} / \mu \mathrm{m}$, such that all co-ions and counterions are removed from the environment of the colloidal particle. To avoid the particle under study drifting towards the electrodes, the particle is optically trapped in the middle between the electrodes [25]. The main difference from previous studies using optical trapping electrophoresis is that we superpose a dc voltage and use a specific suspension of micellar ions to achieve separation of the electrolyte ions. For many colloidal systems, it is practically impossible to separate co-ions and counterions by applying a voltage across a suspension because of fast autodissociation or charge generation. We achieve the separation in a nonpolar colloidal system with charged reverse micelles acting as a micellar-ionic electrolyte, because of its slow generation process [26]. By tuning the applied voltage, the ionic strength of the liquid around the particle can be reduced continuously, essentially to zero, which is the Hückel limit. By comparing the mobility of the particle with a double layer to the mobility of the particle without a double layer in the Hückel limit, this method allows us to investigate the friction or retardation caused by the charge carriers in the double layer. Therefore, this method opens possibilities to verify if the standard theory of electrophoretic retardation applies to nonpolar colloids with charged reverse micelles.

In our theoretical interpretation of the results, a few assumptions are made-for example, that the particle charge remains constant during the time scale of the experiment. Henry's formula for a symmetrical double layer bridging the Hückel and Smoluchowski limits is used for modeling the mobility as a function of the ionic strength, knowing that for some dc fields the double layer is probably asymmetrical. And, we assume that at high dc voltages all co-ions and counterions are stripped from the particle, whereas some charges may remain near the particle. The consequences of these assumptions are elaborated in the discussion section.

With these assumptions, it appears that there is a good qualitative agreement between our experiments and the standard theory for electrophoresis, even though the retardation force appears to be slightly weaker than theoretically expected. Besides measuring fundamental properties of electrophoretic retardation in nonpolar colloids, this method allows us to study the still not fully understood charging mechanisms of nonpolar colloids [27,28] and is relevant for industrial applications such as liquid toner, paint, motor oil additives, and electrophoretic ink displays [29,30].

\section{MATERIALS AND SETUP}

In our experiments, spherical polystyrene divinylbenzene particles (Micropearl SP particles from Sekisui) with radius $R=3.38 \mu \mathrm{m}$ are dispersed at volume fractions below 0.0001 in mixtures of the nonpolar solvent n-dodecane (Rectapur, VWR) and the surfactant polyisobutylene-succinimide (PIBS) (OLOA 11000, Chevron Oronite). The measurements are carried out on individual particles to eliminate errors due to variations in particle size and charge. Above the critical micelle concentration at a surfactant weight fraction around $\phi_{m}=$ 0.00005 , the PIBS surfactant molecules in dodecane form reverse micelles with a hydrodynamic radius of 5 to $7 \mathrm{~nm}$, of which a fraction of about $4 \%$ are positively or negatively charged [31]. The concentration of charged surfactant monomers is negligible compared to the concentrations of positively and negatively charged micelles. Many properties of PIBS surfactant in dodecane, such as conductivity, concentration, and mobility of the charge carriers, have been studied before using sensitive electric current measurements [26,31-34]. From these current measurements, it was concluded that the concentrations of positively and negatively charged reverse micelles are the same $\left(n^{+}=\right.$ $n^{-}=\bar{n}$ ), such that the charged micelles can be seen as micellar ions of a 1:1 electrolyte. Also, the charged reverse micelles form a double layer next to charged electrode surfaces, in good agreement with a simple diffuse double-layer model conform to the solution of the Poison-Nernst-Planck equation and without Stern or Helmholtz layers. Also, measurements of the interaction potential between Poly(methyl methacrylate) particles in dispersions with PIBS surfactant are explained well with this simple double-layer model [11,12]. Therefore, we assume that in the absence of a dc field, the charged particles in this study can be described well with this simple double-layer model and the expressions of Hückel, Smoluchowski, and Henry.

In a pure nonpolar liquid, particle charging occurs at a high energy cost, and particle charges are in the order of a few elementary charges $[28,35]$. The presence of reverse micelles reduces this energy cost considerably and leads to charged colloid systems with surprisingly high particle charges [30]. The actual charging mechanism in this type of nonpolar colloidal dispersion with reverse micelles is still strongly researched by several authors $[11,12,27,36]$. We assume that the particles in this work become negatively charged by dissociation of protons that are encapsulated by a reverse micelle or by preferential adsorption of negatively charged micelles [27]. Four particle dispersions are prepared with surfactant weight fractions $\phi_{m}=0$, $0.0001,0.0004$, and 0.001 . The equilibrium concentration of charged reverse micelles for the mixtures are, respectively, $\bar{n}=0,0.4,2.3$, and $5.3 \mu \mathrm{m}^{-3}$, as determined by electrical transient-current measurements [26], following the trend $\bar{n}=5300 \times \phi_{m} \mu \mathrm{m}^{-3}$. The characteristic thickness of the double layer, the Debye length, is calculated as $\kappa^{-1}=\sqrt{\varepsilon_{0} \varepsilon_{r} k T / 2 e^{2} \bar{n}}$, with dielectric constant $\varepsilon_{0} \varepsilon_{r}$, thermal energy $k T$, and elementary charge $e$. The $\kappa$ values corresponding to the four surfactant concentrations are 0 , $5 \times 10^{5}, 1.3 \times 10^{6}$, and $1.9 \times 10^{6} \mathrm{~m}^{-1}$. The $\kappa R$ values 
corresponding to the different surfactant concentrations are then $\kappa R=0,1.8,4.3$, and 6.5 , respectively. The particle concentration is sufficiently low (below $0.01 \mathrm{vol}$ $\%$ ) that the bulk micelle concentration and properties are not influenced and that we can neglect the contribution of the particles in the calculation of the Debye length. The micelle mobility obtained from transient-current measurements is (in absolute value) $\mu_{\mathrm{CM}}=1200 \mu \mathrm{m}^{2} / \mathrm{Vs}$, corresponding to a Stokes radius of $5 \mathrm{~nm}$ and a diffusion coefficient $D_{\mathrm{CM}}=31 \mu \mathrm{m}^{2} / \mathrm{s}$.

The colloidal mixtures are inserted between indium-tinoxide-covered glass substrates, separated by a distance $d$ of approximately $50 \mu \mathrm{m}$ using UV curable glue containing glass spacers (see Fig. 1). A 975-nm laser beam of approximately $100 \mathrm{~mW}$ is focused with a $100 \times$ oil immersion objective (NA 1.3), and it is used to optically trap a single particle with refractive index 1.57 at the midplane between the electrodes, with trapping stiffness $k_{x}=k_{y}=$ $18 \mathrm{pN} / \mu \mathrm{m}$ in the radial directions and $k_{z}=3 \mathrm{pN} / \mu \mathrm{m}$ in the axial direction. A Nikon Eclipse Ti microscope is used together with an Andor EM-CCD camera for particle imaging.

Image analysis is used to measure the $x, y, z$ position of the particle with sample frequency $50 \mathrm{~Hz}$. The $(x, y)$ position of a single, isolated particle is calculated from each image with $20-\mathrm{nm}$ precision, as the intensity centroid $(x, y)=\sum\left(x_{i}, y_{j}\right)\left(I_{i, j}-I_{b}\right)^{2} / \sum\left(I_{i, j}-I_{b}\right)^{2}$, where the summation goes over pixels with intensity $I_{i, j}$ and with background intensity $I_{b}$ [37]. The normalized radial intensity profile averaged over eight equiangular orientations $I(r)=\left[\left\langle I_{i}(r)\right\rangle-I_{b}\right] / I_{b}$ is calculated, where $r$ is the radial distance to the particle center $(x, y)$. The $z$ position is obtained by comparing $I(r)$ with a calibrated z-stack of linearly spaced normalized intensity profiles (see Fig. 2) using a least-squares fitting procedure with quadratic interpolation. The z-stack itself is generated for each particle individually to achieve an accuracy of approximately $20 \mathrm{~nm}$ in the $z$ direction. First, a sequence of constantvelocity trajectories is acquired in a $\pm 10-\mu \mathrm{m}$ region of interest above and below the focal depth. This is achieved

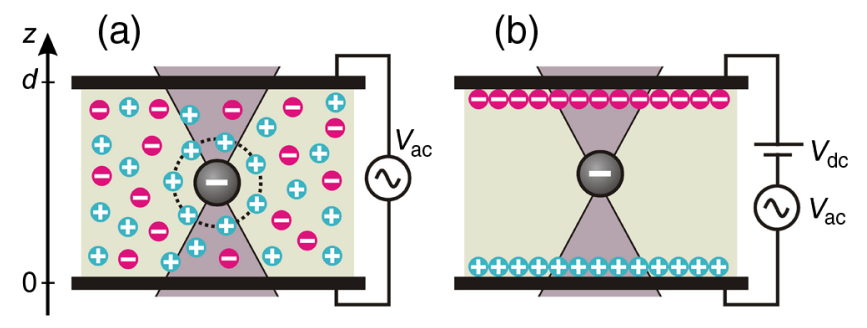

FIG. 1. (a) Schematic representation of a colloidal particle optically trapped at the midplane between two electrodes. The electrophoretic mobility is measured by applying an ac voltage $V_{\text {ac }}$. In (b) the positively and negatively charged reverse micelles are completely or partially separated depending on the magnitude of the additional dc voltage $V_{\mathrm{dc}}$. with a small square wave voltage superposed on a dc voltage compensating for the gravitational force on the particle. Each radial intensity profile is then correlated to a time with respect to a reference start position $z_{\text {ref }}$. By averaging out these times, the effect of random Brownian motion is reduced. As a result, a linear z-stack is constructed, which means that every profile corresponds to a $z$ position of $z_{i}=z_{\text {ref }}+i \Delta z$. It has been verified that the $\mathrm{z}$-stack is, in fact, linear by measuring the linear trajectories of particles falling under the influence of gravity. The values of $z_{\text {ref }}$ and $\Delta z$ are calibrated using a second z-stack in which the particle is immobilized on the bottom substrate. This z-stack is created with 200 radial intensity profiles every $100 \mathrm{~nm}$, by automatically changing the focal depth of the objective stepwise. Even though the presence of the glass substrate gives rise to small aberrations compared to a particle floating in the liquid, the correlation between the two z-stacks is sufficient to calculate $z_{\text {ref }}$ and $\Delta z$. This three-dimensional particle-tracking method allows us to follow particles over a range of tens of micrometers in any direction without needing to readjust the focus.

In the actual experiment, the $z$ position of the particle is monitored while a sinusoidal voltage is applied $\left(V_{\mathrm{ac}}=\right.$ $10 \mathrm{Vpp}, f=21 \mathrm{~Hz}$ ). A dc voltage $V_{\mathrm{dc}}$ is superposed, which increases from $0 \mathrm{~V}$ to a maximum of $2 \mathrm{~V}$ in steps of $0.2 \mathrm{~V}$ after every time interval $\Delta t=4 \mathrm{~s}$. The time scale of the ac mobility measurement $(50 \mathrm{~ms})$ is much smaller than the time scale (about $10 \mathrm{~s}$ ) of changing concentrations of charged reverse micelles around the particle from the dc voltage. Figure 3 shows that the mobility measurement at $10 \mathrm{Vpp}$ and at the sine frequency $f=21 \mathrm{~Hz}$ is in the linear regime. For ac voltages above $20 \mathrm{Vpp}$, nonlinear effects, possibly including the Stotz-Wien effect, cause the

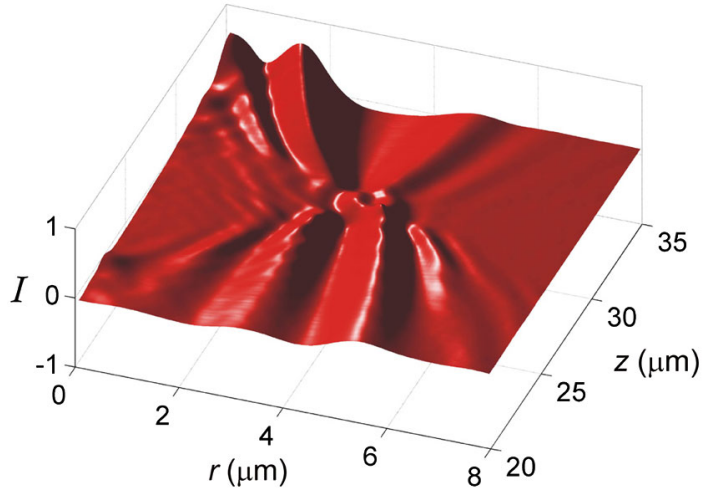

FIG. 2. Surface plot of the normalized radial intensity profile of a spherical particle as a function of the particle position relative to the bottom substrate. The focal depth of the microscope is fixed at $z=28 \mu \mathrm{m}$. By generating a $z$-stack fingerprint of each particle and using a least-squares-fitting procedure, the particle position can be determined with $20-\mathrm{nm}$ precision over a range of tens of micrometers in any direction without needing to readjust the focus. 


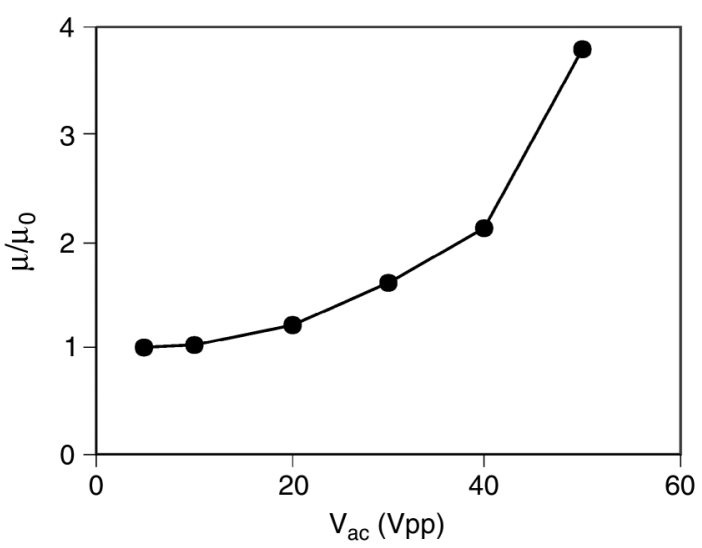

FIG. 3. Electrophoretic mobility values of a single particle with radius $3.38 \mu \mathrm{m}$ in dodecane with surfactant concentration $\phi_{m}=0.001$ for different applied ac voltages $V_{\text {ac }}$, normalized to the value $\mu_{0}$ at $V_{\mathrm{ac}}=5 \mathrm{Vpp}$. The sinusoidal voltage $V_{\mathrm{ac}}$ at frequency $f=21 \mathrm{~Hz}$ is applied across the electrodes separated by $50 \mu \mathrm{m}$ in the absence of a dc voltage. Below $V_{\mathrm{ac}}=10 \mathrm{Vpp}$, the mobility is in the linear regime, while for voltages above $20 \mathrm{Vpp}$, the Stotz-Wien effect and other nonlinear effects start to play a role.

increase in mobility. For sufficiently low applied ac voltages, the time $\tau=d / \kappa\left|\mu_{\mathrm{CM}}\right| V$ for a charged reverse micelle to drift across the double layer because of the externally generated electric field $E=V / d$ is much longer than the relaxation time of the double layer $\tau_{\mathrm{DL}}=$ $1 / \kappa^{2} D_{\mathrm{CM}}$ [38]. The oscillation amplitude of charged micelles from the ac field is $1 \mu \mathrm{m}$, much less than the thickness of the device $(50 \mu \mathrm{m})$. The optical trap is used

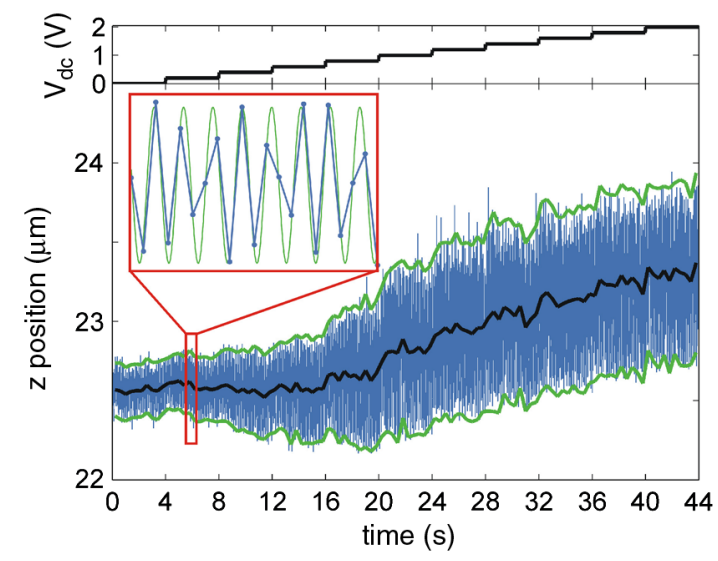

FIG. 4. Measurement of the $z$ position of a particle with radius $3.38 \mu \mathrm{m}$ in a micellar-ionic electrolyte containing surfactant OLOA 11000 with mass fraction $\phi_{m}=0.0004$ in dodecane, sampled at $50 \mathrm{~Hz}$. The particle is optically trapped in the $z$ direction with trapping constant $k_{z}=3 \mathrm{pN} / \mu \mathrm{m}$, while a sinusoidal voltage with $f=21 \mathrm{~Hz}$ and $V=10 \mathrm{Vpp}$ superposed with a stepwise increasing dc voltage $V_{\mathrm{dc}}$ is applied. For increasing dc voltage, the oscillation amplitude increases, and the average position increases as the shifting balance between gravitational, optical, and electrical forces pushes the particle upwards. The inset illustrates the fitting of a sinus to the data. to keep the particle in the middle of the device, despite the effects of gravity, flow, and the dc electric force. Figure 4 illustrates the measurement for the case $\phi_{m}=0.0004$. The oscillation amplitude is determined as a function of time using a sine-fitting algorithm. It is observed that for increasing dc voltage, the oscillation amplitude increases and the average position in the trap shifts because the equilibrium between the gravitational, optical, and electrical forces changes. After every dc-voltage step, the average position and oscillation amplitude increase to their new equilibrium values in about $2 \mathrm{~s}$, such that the equilibrium values can be determined for every value of $V_{\mathrm{dc}}$.

\section{RESULTS}

In Fig. 5, the steady-state electrophoretic mobility is calculated for every value of $V_{\mathrm{dc}}$ and for increasing surfactant concentration using $\mu=2 \pi A f d / V$, where $V=$ $5 \mathrm{~V}$ is the ac-voltage amplitude and $A$ is the oscillation amplitude of the $z$ position. Because the oscillation frequency $f=21 \mathrm{~Hz}$ is higher than the optical-trap corner frequency $f_{z}=k_{z} /\left(12 \pi^{2} \eta R\right.$ ) of about $6 \mathrm{~Hz}$ (where $\eta=$ $1.38 \mathrm{mPas}$ is the dynamic viscosity of dodecane), the optical trap has a negligible influence on the obtained mobility values. The mobility for $V_{\mathrm{dc}}=0 \mathrm{~V}$ corresponds to the classical mobility $\mu_{0}$, for which the double layer is symmetrical and for which there is a homogeneous distribution of charged micelles with concentration $\bar{n}$ in the bulk [see Fig. 1(a)]. The value of $\mu_{0}$ for the measured particles depends on the surfactant concentration, as shown in Fig. 6. When no surfactant is present in the mixture $\left(\phi_{m}=0\right)$, the particle charge is, on average, very small because of the high energetic penalty for the separation of oppositely

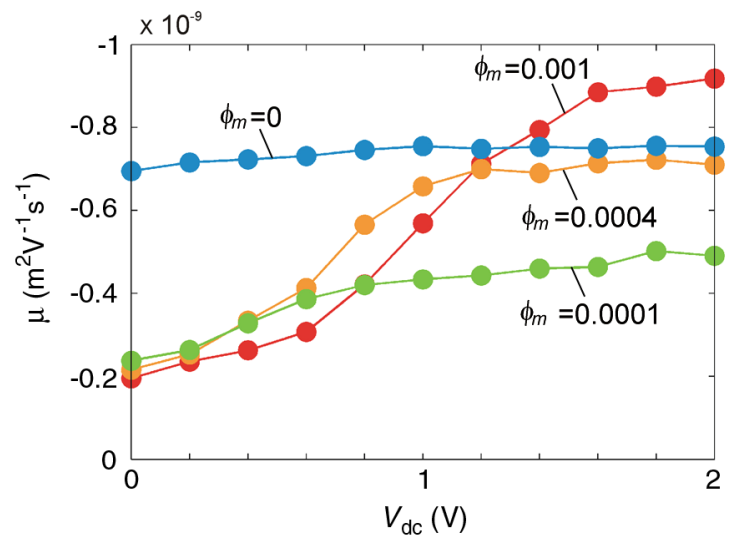

FIG. 5. Mobility measurement of four particles of $3.38 \mu \mathrm{m}$ radius optically trapped in the midplane between the electrodes, for different surfactant concentrations $\phi_{m}$ and different applied voltages $V_{\mathrm{dc}}$. In the absence of surfactant $\left(\phi_{m}=0\right)$, the mobility is independent of the increasing dc voltage. When the solution contains surfactant, the mobility increases for increasing values of $V_{\mathrm{dc}}$, because the micellar ions or charged reverse micelles are gradually separated, thereby reducing the electrophoretic retardation. The saturation value is reached for values of $V_{\mathrm{dc}}$ around $2 \mathrm{~V}$. 


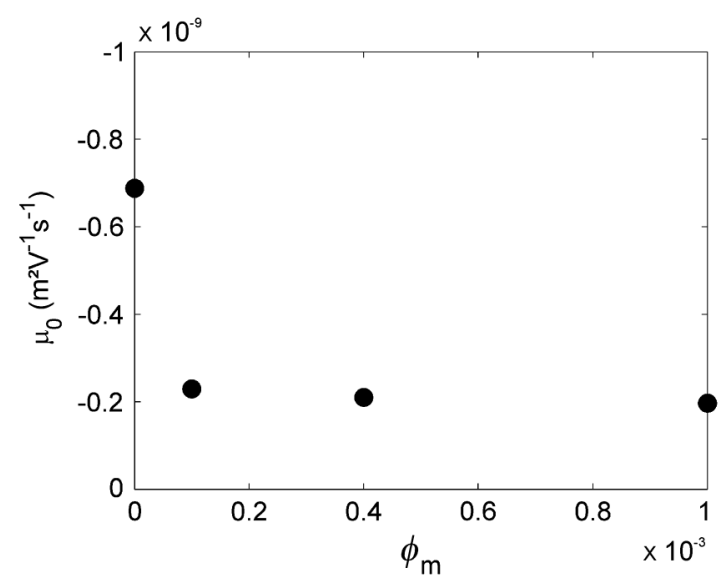

FIG. 6. The equilibrium mobility $\mu_{0}$ is the mobility measured in absence of a dc voltage. Without surfactant $\left(\phi_{m}=0\right)$ the mobility is on average very small, but some particles (as in this case) get a larger charge after contact with a charged electrode. When surfactant is present, the mobility is about $-210 \mu \mathrm{m} \mathrm{V}^{-1} \mathrm{~s}^{-1}$ and is practically independent of the surfactant concentration.

charged ions. But some particles (like the particle measured here) have a much higher charge after touching a charged electrode surface. When surfactant is added, the particles tend to have a well-defined mobility of around $-210 \mu \mathrm{m}^{2} \mathrm{~V}^{-1} \mathrm{~s}^{-1}$, independent of the surfactant concentration. Here, a particle with mobility $-210 \mu \mathrm{m}^{2} \mathrm{~V}^{-1} \mathrm{~s}^{-1}$ corresponds to a zeta potential of about $-25 \mathrm{mV}$. For

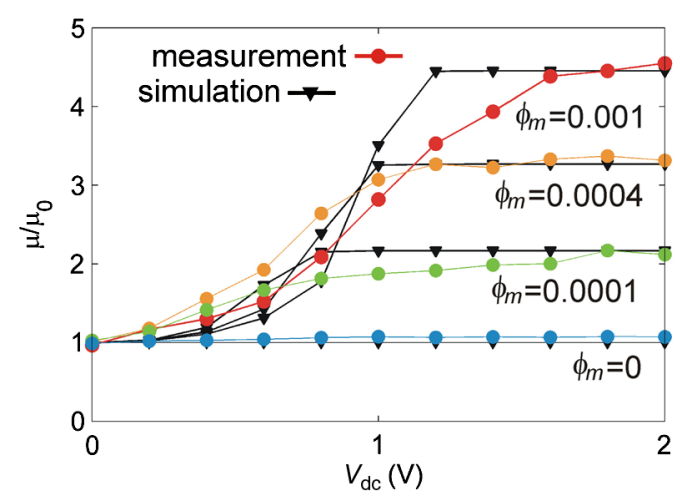

FIG. 7. Measurements $(\bullet)$ and simulations $(\boldsymbol{\nabla})$ of the normalized mobility of $3.38-\mu \mathrm{m}$ radius particles optically trapped in the midplane between the electrodes, as a function of the surfactant concentration $\phi_{m}$ and of the applied dc voltage $V_{\mathrm{dc}}$. For increasing values of $V_{\mathrm{dc}}$, the micellar ions are increasingly separated, which reduces the electrophoretic retardation and increases the mobility. The saturation value is reached for values of $V_{\mathrm{dc}}$ around $2 \mathrm{~V}$. The simulations are based on the standard theory of electrophoresis and on calculations of the local ionic strength, using the best-fitted relation $\bar{n}=2000 \times \phi_{m} \mu \mathrm{m}^{-3}$ for the equilibrium concentration of micellar ions. Similar to the experiments, the simulations are carried out with a 4-s delay between the dc voltage steps.

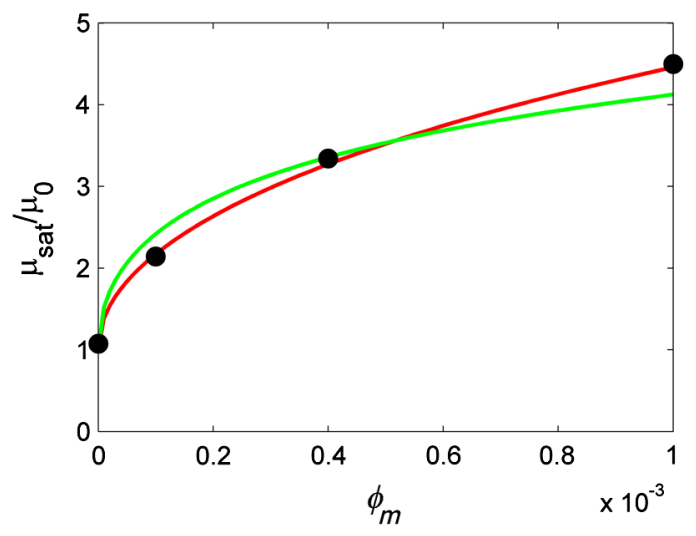

FIG. 8. Measurement $(\bullet)$ of the saturated mobility $\mu_{\text {sat }}$ at $V_{\mathrm{dc}}=2 \mathrm{~V}$ normalized to the equilibrium mobility $\mu_{0}$ at $V_{\mathrm{dc}}=$ $0 \mathrm{~V}$, as a function of the surfactant concentration $\phi_{m}$. The red line shows the expectancy from the standard theory, $\mu_{\text {sat }} / \mu_{0}=$ $[1+\kappa(\bar{n}) R] / f[\kappa(\bar{n}) R]$, using the relations $\bar{n}=2000 \times$ $\phi_{m} \mu \mathrm{m}^{-3}$ and $R=3.38 \mu \mathrm{m}$. For the green line, the retardation force is reduced with a correction factor $\xi=0.9$ while using the concentrations $\bar{n}=5300 \times \phi_{m} \mu \mathrm{m}^{-3}$ obtained from current measurements and $R=3.38 \mu \mathrm{m}$.

better comparison, all mobilities from Fig. 5 are normalized to the corresponding value $\mu_{0}$ in Fig. 7.

In the measurements of Fig. 7 for the dispersions with $\phi_{m}=0.0001,0.0004$, and 0.001 corresponding to $\kappa R=$ $1.8,4.3$, and 6.5 , respectively, the normalized mobility starts increasing at a characteristic value of $V_{\mathrm{dc}}$ around $0.8 \mathrm{~V}$ and saturates at a value $\mu_{\text {sat }}$ for a sufficiently high value of $V_{\mathrm{dc}}$ around $2 \mathrm{~V}$. No significant change in mobility is observed for the sample with $\phi_{m}=0$, which corresponds to pure dodecane. The fraction $\mu_{\text {sat }} / \mu_{0}$, shown in Fig. 8, is found to be larger with increasing surfactant concentration.

\section{ANALYSIS}

The interpretation of the experimental data can be split up into two parts. First, we investigate how the local ionic strength in the middle of the electrodes where the particle is situated depends on the applied dc voltage, and second, we look at how the particle mobility depends on this local ionic strength.

We start by investigating the influence of the dc voltage on the distribution of charged reverse micelles without considering any colloidal particles or ac field. The distributions of positive and negative micellar ions are calculated by solving the one-dimensional Poisson-Nernst-Planck equations:

$$
\begin{aligned}
\frac{\partial n^{ \pm}}{\partial t} & =D_{\mathrm{CM}} \frac{\partial^{2} n^{ \pm}}{\partial z^{2}}-\frac{\partial}{\partial z}\left(n^{ \pm} \mu_{\mathrm{CM}}^{ \pm} E\right), \\
\frac{\partial E}{\partial z} & =\frac{e\left(n^{+}-n^{-}\right)}{\varepsilon_{0} \varepsilon_{r}} .
\end{aligned}
$$


$n^{+}$and $n^{-}$are the concentrations of positive and negative micellar ions, and $E$ is the electric field; these quantities are all time and position dependent. The time-dependent equation is used here because at dc voltages below $1 \mathrm{~V}$, the steady state is not completely reached in the time $\Delta t=4 \mathrm{~s}$ between consecutive increases of the voltage. Such simulations of the transient dynamics have been used in previous work to reproduce, with high accuracy, the electric currents measured in similar micellar-ionic electrolytes and particle trajectories upon application of a voltage step [26,31-34,39]. Generation and recombination of charged reverse micelles are not included in the simulation because the characteristic generation and recombination time constants (almost equal to $1000 \mathrm{~s}$ ) are much larger than the duration of the experiment [26]. Also, in the simulations, it is assumed that there are no electrochemical reactions with micellar ions at the electrode interfaces. This assumption is based on evidence from transient current measurements that faradaic currents are negligible compared to the ionic current from the micellar ions [26]. Depending on the concentration of charged reverse micelles, the applied dc voltage, and the thickness of the device, different types of switching of charged reverse micelles and colloidal particles can occur. For high voltages $\left(V_{\mathrm{dc}} \gg 1 \mathrm{~V}\right)$ and low concentrations of charged reverse micelles $\left(\bar{n} \ll 0.5 \mu \mathrm{m}^{-3}\right)$, the charges move with constant velocity $v=\mu_{\mathrm{CM}} V / d$ towards the electrodes, where they end up in a narrow distribution at the electrodes. The time scale of the charge separation or transit time is then $\tau_{\text {tr }}=d^{2} /\left|\mu_{\mathrm{CM}}\right| V$. For a device with thickness $50 \mu \mathrm{m}$, as in our experiments, and a dc voltage of $1 \mathrm{~V}$, the time scale is $\tau_{\text {tr }}=2.5 \mathrm{~s}$. For high voltages and high concentrations of charged reverse micelles $(\bar{n} \gg$ $0.5 \mu \mathrm{m}^{-3}$ ), there is a complicated space-charge-limited motion of charges: Screening of the electric field causes the charges to move slowly in the bulk, but once they reach the large space-charge regions near the electrodes, they move much faster. The overall switching time scale can be 10 times larger than in the nonscreening case. In both cases, all charged micelles get fully separated in a steady state. For low voltages $\left(V_{\mathrm{dc}} \ll 1 \mathrm{~V}\right)$, the charges move only a small distance before the electric field gets completely screened. Then, in the bulk, the field becomes zero and the concentration in the bulk stays approximately equal to the equilibrium value. In the ideal case of low voltages and high concentrations, the double layer charges exponentially in time with time constant $\tau_{\mathrm{DL}}=d /(2 \kappa D)$ [33].

Parameters used in the simulations are $d=50 \mu \mathrm{m}$, $\mu_{\mathrm{CM}}= \pm 1200 \mu \mathrm{m} \mathrm{V}^{-1} \mathrm{~s}^{-1}, \quad D_{\mathrm{CM}}=31 \mu \mathrm{m}^{2} \mathrm{~s}^{-1}$. The time interval between consecutive increases of the dc voltage is $\Delta t=4 \mathrm{~s}$, identical to the situation in the experiments. Figure 9 illustrates how the distributions of positively and negatively charged reverse micelles depend on the stepwise increasing dc voltage (in steps of $0.2 \mathrm{~V}$ every $\Delta t=4 \mathrm{~s}$ ) for the case of $\bar{n}=1 \mu \mathrm{m}^{-3}$. For voltages below $0.8 \mathrm{~V}$, the electric field is screened by thin space-charge

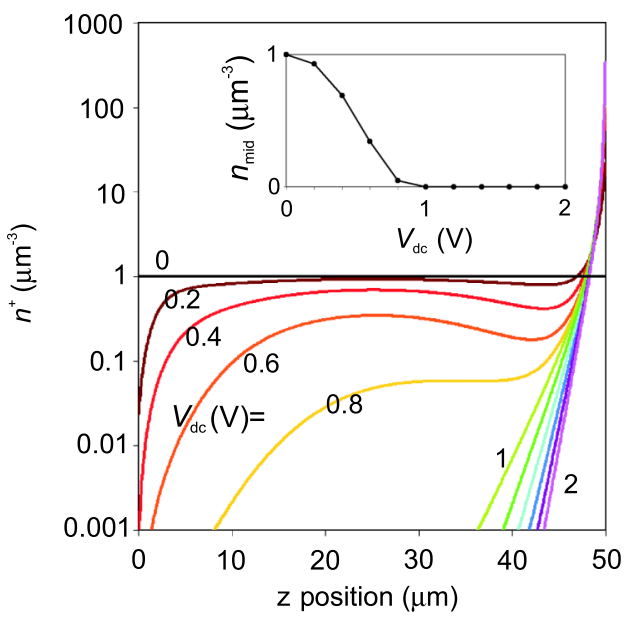

FIG. 9. Simulation of the concentration distribution of positively charged micelles (similar for negatively charged micelles) for $\bar{n}=1 \mu \mathrm{m}^{-3}$ while the voltage $V_{\mathrm{dc}}$ is increased stepwise by $0.2 \mathrm{~V}$ every $4 \mathrm{~s}$. The concentration profiles are shown at the end of each 4-s time interval. The electrodes are situated at $z=0$ and $50 \mu \mathrm{m}$. For voltages below $1 \mathrm{~V}$, the concentration profiles are not in the steady state. At voltages below $0.2 \mathrm{~V}$, the bulk concentration remains approximately the same as $\bar{n}$. For voltages around $0.8 \mathrm{~V}$, the concentrations of positively and negatively charged micelles in the middle become much lower. Above $1 \mathrm{~V}$, the micellar ions are fully separated. In the inset, the concentration $n_{\text {mid }}$ of positively and negatively charged micelles in the midplane between the electrodes (at $z=25 \mu \mathrm{m}$ ) is shown as a function of $V_{\mathrm{dc}}$.

layers at the electrodes, such that the bulk field is zero and the bulk concentration is approximately the same as the equilibrium concentration. Around $0.8 \mathrm{~V}$, the charges become increasingly separated, such that the concentration of micellar ions in the middle drops quickly (see inset in Fig. 9). At about $2 \mathrm{~V}$, the positive and negative charges are completely separated.

With the simulations, the concentrations of micellar ions and the ionic strength near the particle are determined for the different equilibrium concentrations of micellar ions and for each value of the increasing dc voltage. We assume that the presence of a colloidal particle has no influence on the ionic strength in the middle of the device. We also assume that the concentration of charged reverse micelles varies sufficiently slowly in the neighborhood of the particle, such that the particle can be assumed to be situated in a homogeneous electrolyte with bulk concentration $n_{\text {mid }}$.

Next, the dependency of the mobility on the local ionic strength is calculated with the standard formula for the electrophoretic mobility, which takes the effects of electrophoretic retardation into account $[8,40]$ :

$$
\mu=\frac{Q}{6 \pi \eta R\left[1+\kappa\left(n_{\mathrm{mid}}\right) R\right]} f\left[\kappa\left(n_{\mathrm{mid}}\right) R\right],
$$

with $Q$ the bare particle charge, which is assumed to be constant, and $n_{\text {mid }}$ the concentration in the middle between 
the electrodes, where the particle is situated. The notation $\kappa\left(n_{\text {mid }}\right)$ in Eq. (2) emphasizes the dependency of the inverse of the Debye length on the concentration $n_{\text {mid }}$. By normalizing the mobility values to the equilibrium mobility $\mu_{0}$, for which $n_{\text {mid }}=\bar{n}$, the comparison between particles with different bare charge and between measurements and theory is simplified. We note that Eq. (2) is valid for a symmetrical double layer. We expect that this assumption will lead to small errors for the intermediate dc voltages around $1 \mathrm{~V}$, where the external field becomes larger than the field of the double layer. For voltages around $2 \mathrm{~V}$, the double layer is stripped off, such that the problem of asymmetry disappears and the mobility of a point charge is again well described by Eq. (2). The factor $f(\kappa R)$ is approximated here by Henry's expression [5,8], but since its value in our experiments is between 1 and 1.19 , this factor is not very relevant.

For sufficiently high dc voltages, $n_{\text {mid }}$ becomes zero, which is the Hückel limit. Then, the particle potential is not screened anymore, and the mobility becomes that of a point charge:

$$
\mu_{\mathrm{sat}}=\frac{Q}{6 \pi \eta R} .
$$

Combining Eqs. (2) and (3), the fraction $\mu_{\text {sat }} / \mu_{0}$ is theoretically expected to be

$$
\frac{\mu_{\mathrm{sat}}}{\mu_{0}}=\frac{1+\kappa(\bar{n}) R}{f(\kappa(\bar{n}) R)}
$$

since $n_{\text {mid }}$ equals $\bar{n}$ at $V_{\mathrm{dc}}=0 \mathrm{~V}$.

From the experimental values of $\mu_{\text {sat }}$, the bare charge can be derived using $Q=6 \pi \eta R \mu_{\text {sat }}$, following from Eq. (3), corresponding typically to 400 elementary charges. The retardation force $F_{\text {ret }}$ can be calculated using the force balance on the colloidal particle: $Q E=6 \pi \eta R \mu E+F_{\text {ret }}$. This force balance states that the electrostatic force $Q E$ is counteracted by hydrodynamic friction $\left(F_{\text {friction }}=6 \pi \eta R v\right.$ with $v=\mu E$ ) and the retardation force $F_{\text {ret }}$. Combining this result with Eq. (3), we find

$$
F_{\text {ret }}=6 \pi \eta R E\left(\mu_{\text {sat }}-\mu\right) \text {. }
$$

Some typical values for the retardation force at field strength $E=10^{5} \mathrm{~V} / \mathrm{m}$ are $0,2,5$, and 19 piconewton for the four particles in Fig. 5 at $V_{\mathrm{dc}}=0 \mathrm{~V}$. The dependency of the retardation force on the ionic strength is clearer when we rewrite the force balance $Q E=6 \pi \eta R \mu E+$ $F_{\text {ret }}$ using Eq. (2):

$$
F_{\text {ret }}=Q E\left(1-\frac{f\left[\kappa\left(n_{\mathrm{mid}}\right) R\right]}{1+\kappa\left(n_{\mathrm{mid}}\right) R}\right) .
$$

Equation (6) can be derived alternately by calculating the volume force on the diffuse part of the double layer and solving the Navier-Stokes equations.

By combining Eqs. (1) and (2), the normalized electrophoretic mobility is obtained as a function of $V_{\mathrm{dc}}$.
However, calculations with parameters $d=50 \mu \mathrm{m}$, $\mu_{\mathrm{CM}}= \pm 1200 \mu \mathrm{m}^{2} \mathrm{~V}^{-1} \mathrm{~s}^{-1}$, and $D_{\mathrm{CM}}=31 \mu \mathrm{m}^{2} \mathrm{~s}^{-1}$, and with the equilibrium charge concentrations according to $\bar{n}=5300 \times \phi_{m} \mu \mathrm{m}^{-3}$, predict mobility increases that are greater than what was observed in the experiments. In Fig. 7, we show that the optimum fit between experiment and theory is obtained by using $\bar{n}=0.2,0.8$, and $2 \mu \mathrm{m}^{-3}$ for the homogeneous initial micellar-ion concentrations, following the trend $\bar{n}=2000 \times \phi_{m} \mu \mathrm{m}^{-3}$, corresponding, respectively, to $\kappa R=1.26,2.53$, and 4.0. This result is 2.6 times lower than the trend $\bar{n}=5300 \times \phi_{m} \mu \mathrm{m}^{-3}$ obtained from current measurements. The fraction $\mu_{\text {sat }} / \mu_{0}$ in Eq. (4) is plotted as a function of $\phi_{m}$ in Fig. 8, again using $\bar{n}=2000 \times \phi_{m} \mu \mathrm{m}^{-3}$, together with the experimental data.

Different assumptions could be at the root of this quantitative difference between experiment and theory. Besides a 2.6 times lower micellar-ion concentration than measured from transient-current measurements, there are also other explanations for the difference between our experiments and the standard theory. In the next section, fast regulation of the particle charge, a weaker hydrodynamic force transfer from the charged reverse micelles to the particle than predicted by the theory, and the possibility that not all coions and counterions are stripped off the particle at high voltages are discussed in detail.

\section{DISCUSSION}

The dependencies of the particle mobility on both $V_{\mathrm{dc}}$ and $\phi_{m}$ largely agree with the analysis based on the standard theory of electrophoretic retardation, together with calculations of the Nernst-Planck-Poisson equations. The mobility is found to increase around $V_{\mathrm{dc}}=0.8 \mathrm{~V}$, where the concentrations of micellar ions near the particle quickly decrease. This observation can be understood from the diminishing retardation force caused by co-ions and counterions in the diffuse double layer around the particle. The fact that the mobility reaches a saturation value at $V_{\mathrm{dc}}=2 \mathrm{~V}$, where the micellar ions become fully separated, can be understood since the retardation force then becomes zero. By further increasing the dc voltage, the retardation force remains zero, and the mobility does not increase further. The fact that the fraction $\mu_{\text {sat }} / \mu_{0}$ increases for an increasing surfactant weight fraction $\phi_{m}$ can be understood, since the equilibrium concentration of micellar ions $\bar{n}$ increases proportionally to $\phi_{m}$ and because the retardation force is stronger for higher ionic strengths [see Eq. (6)].

The deviations of the experiments from the standard theory may be due to limiting assumptions in the theory that are difficult to verify. First, the interpretation of the experimental results is based on the assumption that the bare particle charge remains constant during the experiment. The observation of no significant hysteresis in the mobility while increasing and decreasing the dc voltage 
only indicates that the charge indeed remains constant or that charge regulation happens at a much faster time scale than the duration of a mobility measurement. There are two typically studied cases of charge regulation: constant charge and constant potential. The case of constant-charge regulation is indistinguishable from our assumption of a constant bare charge. Only the diminishing retardation force can then explain the increasing mobility for increasing dc voltages. The case of constant potential $\Phi$ corresponds to a practically constant mobility $\mu=\Phi \varepsilon_{0} \varepsilon_{r} / 3 \eta$ [when ignoring the factor $f(\kappa R)$ ], which is not compatible with our observations. However, intermediate cases between constant-charge and constant-potential regulation exist for which the fraction $\frac{\mu_{\text {sat }}}{\mu_{0}}=\frac{Q_{\text {sat }}}{Q_{0}} \frac{(1+\kappa R)}{f(\kappa R)}$ becomes dependent on the fraction of the bare charge $Q_{\text {sat }}$ when high dc voltage around $2 \mathrm{~V}$ is applied and the equilibrium bare charge $Q_{0}$ when no dc voltage is applied. Then, when interpreting $\mu_{\text {sat }} / \mu_{0}$ while assuming a constant bare charge, the retardation force can be underestimated. Second, we assume that at dc voltages around $2 \mathrm{~V}$, all coions and counterions are stripped off the particle surface. Considering the strong electrostatic force between opposite charges in nonpolar media, it is possible that a fraction of the charged reverse micelles remain near the particle surface and reduce the effective particle charge. The saturation value of the mobility is then lower than theoretically expected, leading to $\frac{\mu_{\text {sat }}}{\mu_{0}}=\frac{1+\kappa R}{1+\kappa_{\text {sat }} R}$, where $\kappa_{\text {sat }}$ characterizes the residual charges in the double layer. Third, we expect small errors in the theory for intermediate dc voltages ( $V_{\mathrm{dc}} \cong 1 \mathrm{~V}$ ) due to the asymmetry of the double layer, which is not included in Henry's formula. This asymmetry does not change the fraction $\mu_{\text {sat }} / \mu_{0}$ and can therefore not explain why these values are lower than expected by the theory. Fourth, the electrophoretic retardation force may also be reduced for the present case of large micellar ions instead of the usual molecular ions. By introducing a small correction factor $\xi$ for the retardation force, the experimental observations can be explained as well. With this correction factor, the force balance on the particle becomes $Q E=6 \pi \eta R \mu E+\xi F_{\text {ret }}$, where $F_{\text {ret }}$ is still the retardation force as described by the standard theory. Now, the ratio between the saturation and equilibrium values of the mobility becomes $\frac{\mu_{\text {sat }}}{\mu_{0}}=\frac{1}{\left[1+\xi\left(\frac{f(\kappa R)}{1+\kappa R}-1\right)\right]}$, which is plotted in Fig. 8 as well, using the ion concentrations following $\bar{n}=$ $5300 \times \phi_{m} \mu \mathrm{m}^{-3}$ obtained from current measurements as known quantities and using $\xi=0.9$. The consequences of these four assumptions remain to be studied in more detail to achieve a more reliable measurement of the retardation force.

The demonstration of a dependence of the particle mobility on the local micellar-ion concentration also sheds light on previously unexplained particle acceleration near the attracting electrode of in-plane electronic ink devices [41]. There, the micellar ions are already separated before the particles have reached the electrodes, since the mobility of colloidal particles is smaller than that of the micellar ions. As shown in the present paper, the absence of micellar ions can explain an increase in particle mobility and, therefore, the anomalous acceleration near the electrodes. The method also opens possibilities to study charging mechanisms of colloidal particles in nonpolar media, since the effect of changing concentrations of co-ions and counterions on the particle charge can be studied in a direct way on single particles.

\section{CONCLUSIONS}

We have demonstrated a method to investigate the electrophoretic retardation effect of single colloidal particles in a nonpolar micellar 1:1 electrolyte. By applying a small dc voltage, the local ionic strength near the particle can be reduced, essentially to zero. This method avoids the extremely high fields used in measurements based on the Stotz-Wien effect, in which co-ions and counterions are still present around the particle, but the formation of the double layer is inhibited. The method allows us to measure the bare particle charge and the retardation force acting on the particle. In such a way, the standard theory of electrophoresis can be verified for nonpolar colloids with a micellar-ionic electrolyte. Qualitatively, the measurements of the electrophoretic mobility as a function of the applied dc voltage and of the equilibrium concentration of micellar ions are in good agreement with calculations based on the standard theory of electrophoresis and simulations of the micellar-ion concentration using the Nernst-PlanckPoisson equations.

\section{ACKNOWLEDGMENTS}

This research was supported by the Research Foundation-Flanders (FWO Vlaanderen), IWTVlaanderen, the IAP-VI project photon@be funded by BELSPO, the Belgian Science Policy program and the Hercules Foundation (Project No. AUGE/013).

[1] J. Lyklema, Fundamentals of Interface and Colloid Science, Solid-Liquid Interfaces, Vol. II (Academia Press, London, 1995).

[2] J.H. Masliyah and S. Bhattacharjee, Electrokinetics and Colloid Transport Phenomena (Wiley, New York, 2006).

[3] E. Hückel, The Cataphoresis of the Sphere, Phys. Z. 25, 204 (1924).

[4] M. von Smoluchowski, Contribution to the Theory of Electro-osmosis and Related Phenomena, Bull. Int. Acad. Sci. Cracovie 3, 184 (1903).

[5] D. C. Henry, The Cataphoresis of Suspended Particles. Part I. The Equation of Cataphoresis, Proc. R. Soc. A 133, 106 (1931).

[6] P. H. Wiersema, A.L. Loeb, and J.Th. G. Overbeek, Calculation of the Electrophoretic Mobility of a 
Spherical Colloid Particle, J. Colloid Interface Sci. 22, 78 (1966).

[7] R. W. O'Brien and L. R. White, Electrophoretic Mobility of a Spherical Particle, J. Chem. Soc., Faraday Trans. 2 74, 1607 (1978).

[8] A. V. Delgado, F. González-Caballero, R. J. Hunter, L. K. Koopal, and J. Lyklema, Measurement and Interpretation of Electrokinetic Phenomena (IUPAC Technical Report), Pure Appl. Chem. 77, 1753 (2005).

[9] H. Ohshima, Theory of Electrostatics and Electrokinetics of Soft Particles, Sci. Tech. Adv. Mater. 10, 063001 (2009).

[10] M.Z. Bazant and T.M. Squires, Induced-Charge Electrokinetic Phenomena, Curr. Opin. Colloid Interface Sci. 15, 203 (2010).

[11] M.F. Hsu, E. R. Dufresne, and D. A. Weitz, Charge Stabilization in Nonpolar Solvents, Langmuir 21, 4881 (2005).

[12] S. K. Sainis, J.W. Merrill, and E. R. Dufresne, Electrostatic Interactions of Colloidal Particles at Vanishing Ionic Strength, Langmuir 24, 13334 (2008).

[13] S. K. Sainis, V. Germain, and E. R. Dufresne, Statistics of Particle Trajectories at Short Time Intervals Reveal fNScale Colloidal Forces, Phys. Rev. Lett. 99, 018303 (2007).

[14] J. W. Merrill, S. K. Sainis, and E. R. Dufresne, Many-Body Electrostatic Forces between Colloidal Particles at Vanishing Ionic Strength, Phys. Rev. Lett. 103, 138301 (2009).

[15] D. Barten, J. M. Kleijn, J. Duval, H.P. v. Leeuwen, J. Lyklema, and M. A. Cohen Stuart, Double Layer of a Gold Electrode Probed by AFM Force Measurements, Langmuir 19, 1133 (2003).

[16] A.S. Dukhin and S.S. Dukhin, Aperiodic Capillary Electrophoresis Method using an Alternating Current Electric Field for Separation of Macromolecules, Electrophoresis 26, 2149 (2005).

[17] M. Wien, Über eine Abweichung vom Ohmschen Gesetze bei Elektrolyten, Ann. Phys. (Berlin) 388, 327 (1927).

[18] J. Lyklema, Fundamentals of Colloid Science: Fundamentals, Vol. I(Academic Press London, London, 1991).

[19] H. Falkenhagen, The Principal Ideas in the Interionic Attraction Theory of Strong Electrolytes, Rev. Mod. Phys. 3, 412 (1931).

[20] C. B. Li and S. P. Friedman, An Apparatus for Measuring the Wien Effect in Suspensions, Colloids Surf. A 222, 133 (2003).

[21] E. Seyrek, P. L. Dubin, and G. R. Newkome, Effect of Electric Field on the Mobility of Carboxyl-Terminated Dendrimers J. Phys. Chem. B 108, 10168 (2004).

[22] S. Stotz, Field Dependence of Electrophoretic Mobility of Particles Suspended in Low-Conductivity Liquids, J. Colloid Interface Sci. 65, 118 (1978).

[23] F. G. Jin, H. T. Davis, D.F. Evans, and R. E. Viturro, Electrophoretic Behaviour in Model Colloidal Systems, in Proceedings of the IS\&T's NIP 14: International Conference on Digital Printing Technologies, Toronto, Canada, 1998 (Springfield, Virginia, 1998), pp. 206-209.

[24] J.C. Thomas, K.L. Hanton, and B. J. Crosby, Measurement of the Field Dependent Electrophoretic Mobility of Surface Modified Silica/AOT Suspensions, Langmuir 24, 10698 (2008).
[25] R. Galneder, V. Kahl, A. Arbuzova, M. Rebecchi, J. O. Rädler, and S. McLaughlin, Microelectrophoresis of a Bilayer-Coated Silica Bead in an Optical Trap: Application to Enzymology, Biophys. J. 80, 2298 (2001).

[26] F. Strubbe, A. R. M. Verschueren, L. J. M. Schlangen, F. Beunis, and K. Neyts, Generation Current of Charged Micelles in Nonaqueous Liquids: Measurements and Simulations, J. Colloid Interface Sci. 300, 396 (2006).

[27] G. S. Roberts, R. Sanchez, R. Kemp, T. Wood, and P. Bartlett, Electrostatic Charging of Nonpolar Colloids by Reverse Micelles, Langmuir 24, 6530 (2008).

[28] F. Beunis, F. Strubbe, K. Neyts, and D. Petrov, Beyond Millikan: The Dynamics of Charging Events on Individual Colloidal Particles, Phys. Rev. Lett. 108, 016101 (2012).

[29] A. R. M. Verschueren et al., Optical Performance of InPlane Electrophoretic Color E-paper, Journal of the SID 18, 1 (2010).

[30] I. D. Morrison, Electrical Charges in Nonaqueous Media, Colloids Surf. A 71, 1 (1993).

[31] F. Beunis, F. Strubbe, M. Marescaux, K. Neyts, and A. R. M. Verschueren, Micellization and Adsorption of Surfactant in a Nonpolar Liquid in Micrometer Scale Geometries, Appl. Phys. Lett. 97, 181912 (2010).

[32] A. R. M. Verschueren, P. H. L. Notten, L. J. M. Schlangen, F. Strubbe, F. Beunis, and K. Neyts, Screening and Separation of Charges in Microscale Devices: Complete Planar Solution of the Poisson-Boltzmann Equation, J. Phys. Chem. B 112, 13038 (2008).

[33] F. Beunis, F. Strubbe, M. Marescaux, J. Beeckman, K. Neyts, and A. R. M. Verschueren, Dynamics of Charge Transport in Planar Devices, Phys. Rev. E 78, 011502 (2008).

[34] K. Neyts, F. Beunis, F. Strubbe, M. Marescaux, B. Verboven, M. Karvar, and A. R. M. Verschueren, Charge Transport and Current in Non-polar Liquids, J. Phys. Condens. Matter 22, 494108 (2010).

[35] F. Strubbe, F. Beunis, and K. Neyts, Detection of Elementary Charges on Colloidal Particles, Phys. Rev. Lett. 100, 21 (2008).

[36] F. Strubbe, F. Beunis, M. Marescaux, and K. Neyts, Charging Mechanism in Colloidal Particles Leading to a Linear Relation between Charge and Size, Phys. Rev. E 75, 031405 (2007).

[37] M. Kvarnström and C. A. Glasbey, Estimation of Centres and Radial Intensity Profiles of Spherical Nano-Particles in Digital Microscopy, Biom. J. 49, 300 (2007).

[38] M. Minor, A. J. van de Linde, H. P. Van Leeuwen, and J. Lyklema, Dynamic Aspects of Electrophoresis and Electroosmosis: A New Fast Method for Measuring Particle Mobilities, J. Colloid Interface Sci. 189, 370 (1997).

[39] F. Strubbe, F. Beunis, M. Marescaux, B. Verboven, and K. Neyts, Electrokinetics of Colloidal Particles in Nonpolar Media Containing Charged Inverse Micelles, Appl. Phys. Lett. 93, 254106 (2008).

[40] M. Evers, N. Garbow, D. Hessinger, and T. Palberg, Electrophoretic Mobility of Interacting Colloidal Spheres, Phys. Rev. E 57, 6774 (1998).

[41] J. Y. Kim, S. Garoff, J. L. Anderson, and L. J. M. Schlangen, Movement of Colloidal Particles in TwoDimensional Electric Fields, Langmuir 21, 10941 (2005). 